\title{
FAILURES OF ADAPTIVE CONTROL THEORY AND THEIR RESOLUTION
}

\author{
BRIAN D. O. ANDERSON* \\ Dedicated to Professor Thomas Kailath on his 70th birthday
}

\begin{abstract}
Adaptive control is a very appealing technology, at least in principle. Yet its use has been conditioned by an attitude of distrustfulness on the part of some practitioners. In this paper, we explain why such distrustfulness is warranted, by reviewing a number of adaptive control approaches which have proved deficient for some reason that has not been immediately apparent. The explanation of the deficiencies, which normally were reflected in unexpected instabilities, is our main concern. Such explanations, coupled with remedies for avoiding the deficiencies, are necessary to engender confidence in the technology. These include the unpredictable failure of the MIT rule; the bursting phenomenon, and how to prevent it; the Rohrs' counterexample, which attempted to disqualify all adaptive control algorithms; the notion that identification of a plant is only valid conceptually for a restricted range of controllers (with the implication that in adaptive control, certain controller changes suggested by adaptive control algorithms may introduce instability); and the concept of multiple model adaptive control.
\end{abstract}

1. Introduction. Adaptive controllers are a fact of life, and have been for some decades. However, theory and practice have not always tracked one another. In this paper, we examine several instances of such a mismatch. These are:

- The MIT rule, an intuitively based gradient descent algorithm that gave unpredictable performance; satisfactory explanation of performance started to become possible in the 1980s.

- Bursting, a phenomenon of temporary instability in adaptive control algorithm implementation of a type observed in the 1970s; explanation and our understanding of avoidance mechanisms only became possible in the 1980s.

- The Rohrs' counterexample, which argued that adaptive control laws existing at the time could not be used with confidence in practical designs, because unmodeled dynamics in the plant could be excited and yield an unstable control system.

- Iterative controller re-design and identification, an intuitively appealing approach to updating controllers that came to prominence in the 1980s and 1990s, and which can lead to unstable performance. Explanation and an understanding of an avoidance mechanism came around 2000.

- Multiple model adaptive control, another intuitively appealing approach to adaptive control with the potential to include non-linear systems. It too can lead to unstable performance; early theoretical development left untouched important issues of the number of controllers to be used, and their location

* National ICT Australia and Research School of Information Sciences and Engineering, Australian National University, Canberra, ACT 0200, Australia. E-mail: brian.anderson@nicta.com.au 
in parameter space. These issues have only been dealt with in the last five years.

The common thread through all these examples except for the Rohrs' counterexample is that algorithms to secure adaptive control were postulated, and the algorithms at times led to instability (though for quite different causes). By instability here, we mean to include the notion of temporary instability; in practical terms, temporary instability occurs when a destabilising controller is connected to a plant for a time interval that is at least as big as a number of time constants of the closed loop system, so that signal levels grow to often destructive levels. In all four situations, this instability was not predicted, and in this sense, the theory behind the algorithms at the time of their advancement (and implicit endorsement by those advancing them) must be regarded as inadequate. It is easy to be a prisoner of one's past and view a new idea through overly optimistic spectacles, and the author is certainly one of those who has done this in the past.

The Rohrs' counterexample could be regarded as constituting an example where instability was present, and an explanation attempted, but the explanation was at the very least highly incomplete.

The examples of a theory-practice mismatch are in fact not the only examples we might have picked on. Let us point out just two more practical difficulties, which most theory has left unaddressed, but which will not be explored further in this paper.

- There are many adaptive control theorems which run along the lines that if conditions A, B and C hold, then all signals in the loop are bounded and some form of convergence occurs. These theorems leave open the theoretical probability of $1 \mathrm{MA}$ current in a $10 \mathrm{~W}$ motor; it is not hard to provide adaptive control simulation examples demonstrating this. The real point is that temporary instability, usually from the temporary insertion of a destabilising controller, is a practically unacceptable phenomenon, but typically not excluded by the theory.

- Much adaptive control theory postulates unknownness, to some degree at least, of the plant, together with a performance index, which should be minimized. Given that the plant is unknown, it may be the case that the performance index, at least in practical terms, can never be minimized. What if for the real unknown plant, a linear-quadratic minimisation were to yield a controller giving rise to a closed-loop phase margin of .01 radians? Any attempt to construct an adaptive controller will founder in tears, or typically instability, no matter what the theory says, and adaptive control theories for the most part sidestep any treatment of this issue.

The structure of the paper is as follows. In Section 2, we present a very high level statement of what constitutes adaptive control. The next five sections treat each of the five problems we first identified above. The final section contains concluding 
remarks.

2. Adaptive Control. Our aim in this section is to summarize material that is now to be found in scores of textbooks and hundreds of papers. We cite a few textbooks $[1,2,3,4,5,6]$. A conventional control loop appears as in Figure 1. The controller is determined using knowledge of the plant together with a list of specifications on the closed-loop performance: the specifications may include the requirement to minimise a certain performance index.

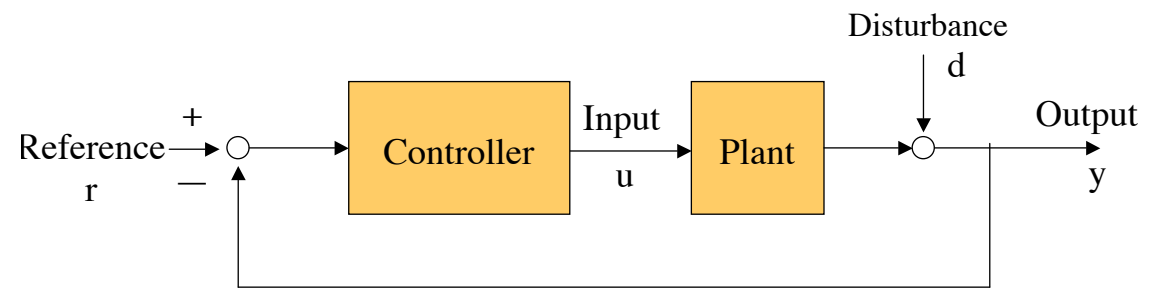

Fig. 1. Conventional Control Loop

The first thing that is different in an adaptive control context is that the plant is initially unknown, or only partially known, or it may be slowly varying. Since in many cases, a single controller will not be able to deliver a satisfactory result for any possible plant, it is necessary to incorporate some learning capability in the controller. One can of course conceive of doing this whether or not the plant and controller are linear; for the most part here, we shall confine consideration to linear plants and controllers. A typical non-adaptive controller maps the error signal $r-y$ of Figure 1 into the plant input $u$ in a causal time-invariant manner:

$$
\begin{array}{r}
\dot{x}_{c}=A_{c} x_{c}+b_{c}(r-y) \\
u=c_{c} x_{c}
\end{array}
$$

where $A_{c}, b_{c}, c_{c}$ are constant matrices, and $x_{c}$ is the state vector of the controller. In an adaptive controller some of the entries of $A_{c}, b_{c}$ or $c_{c}$ are adjusted. One high level architecture is shown in Figure 2

The job of the identifier is to determine what the plant is, or at least to determine an estimate of the plant. The control law calculator does on-line what would be done by the designer before controller introduction in a conventional control problem where the plant is known.

Most of the theory of adaptive control is concerned with writing down algorithms to do these various tasks, and to establishing that the algorithms do what is desired 


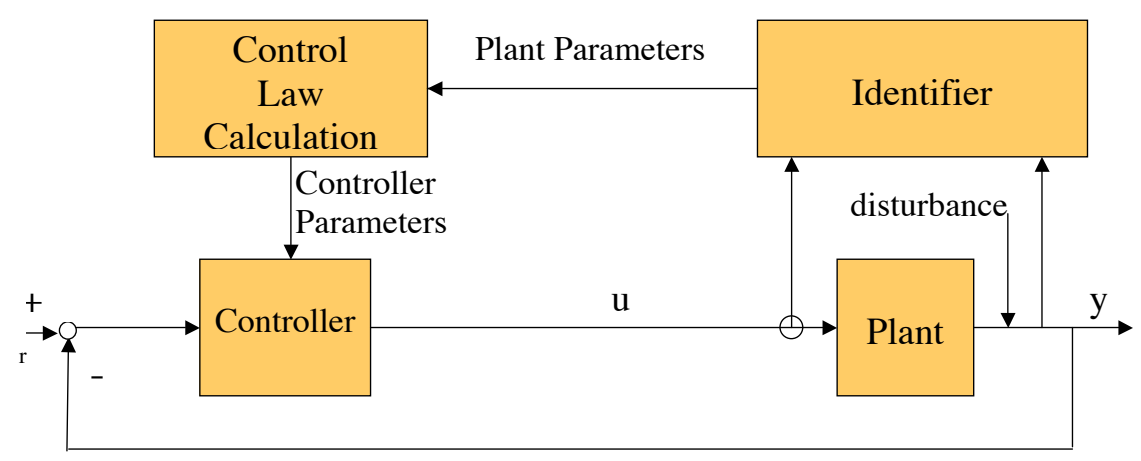

FIG. 2. A high level architecture for adaptive control

with no untoward side effects.

For future reference, we point out the existence of three time scales associated with the scheme of Figure 2:

- The time scale of the underlying closed-loop dynamics, with fixed plant parameters and controller parameters

- The time scale for identifying the plant

- The time scale associated with plant parameter variations

It is clear that the identification time scale needs to be faster than the plant variation time scale, else identification cannot keep up. It also turns out that it is harder to develop good adaptive controllers, which identify (and thus adjust the controllers) at a time scale comparable with that of the closed-loop dynamics. Interaction of the two processes can occur and generate instability. Nevertheless, operational requirements may force comparability of the time scales, for example in the case of a sudden component failure of a plant, where controller reduction must occur very fast to avoid catastrophe; such adaptive control problems are extremely challenging.

By way of more technical comment to those who are not novices in the field of adaptive control, we would suggest that much adaptive control work of the 1980s and 1990s in retrospect can be seen as seeking algorithms in which the two time scales of identification and closed-loop dynamics were comparable; conditions like positive realness of certain transfer functions allow the construction of Lyapunov functions which may establish stability without time scale separation. It is also interesting to note in passing that some attempts to speed up the identification and control time scales to both be fast can result in chaos $[7,8]$.

3. The MIT Rule. The MIT rule is a scalar parameter adjustment law which was proposed around 1960 for the adaptive control of a linear system modelled as a cascade of a linear stable plant and a single unknown gain $[9,10]$. The adjustment 
law involves approximating a gradient-descent procedure seeking the minimum of an integral-squared performance criterion. The initial intended application was to the control of aircraft dynamics where the single unknown parameter was related to dynamic pressure.

In the history of adaptive control, the MIT Rule represented a watershed; it offered the possibility of adaptation for a useful application, the method was simply formulated, and apparently straightforward to apprehend in an intellectual sense. Performance however turned out to be unpredictable; explanations (as opposed to mere reporting of the performance) took some time to be achieved, see e.g. [11].

The basic set up is shown in Figure 3. The plant is $k_{p} Z_{p}(s)$, where $k_{p}$ is unknown apart from its sign, and $Z_{p}(s)$ is a known stable transfer function. The basis of identification is that a cascade adjustable positive known gain $k_{c}(t)$ is introduced as shown, and the output of the upper arm is compared to the output of $k_{m} Z_{p}(s)$ when the same driving signal is applied; here, $k_{m}$ is a known gain, with the same sign as $k_{p}$. Of course, if $k_{p} k_{c}(t)=k_{m}$ for all $t$, zero error will result and $k_{p}$ will be given as $k_{m} / k_{c}$. If the error $e(t)$ is nonzero the idea is to adjust $k_{c}$ to cause it to go to zero. The MIT rule is the rule of adjustment for $k_{c}$.

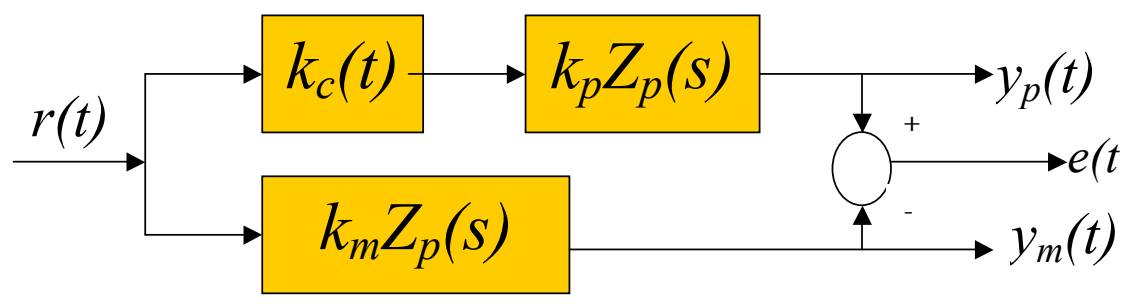

FIG. 3. Basic Set-Up for MIT Rule

The idea is to use gradient descent, i.e.,

$$
\dot{k}_{c}=-g \frac{\partial}{\partial k_{c}}\left[\frac{1}{2} e^{2}(t)\right]
$$

where $g$ is a positive gain constant. Equivalently,

$$
\dot{k}_{c}=-g\left[y_{p}-y_{m}\right] y_{m} .
$$

Sometimes this works, and sometimes it does not work. Figure 4 shows the result for a plant $Z_{p}(s)=(s+1)^{-1}$, with unit amplitude sinusoidal input of frequency $\omega$, and variable adaptive gain $g$. How can one explain the instability?

Broadly speaking, the instability being displayed is a result of interaction of the adaptive loop dynamics with the plant dynamics, a phenomenon that is occurring when the time scales are comparable, but does not occur otherwise. We shall explain this in more detail. 


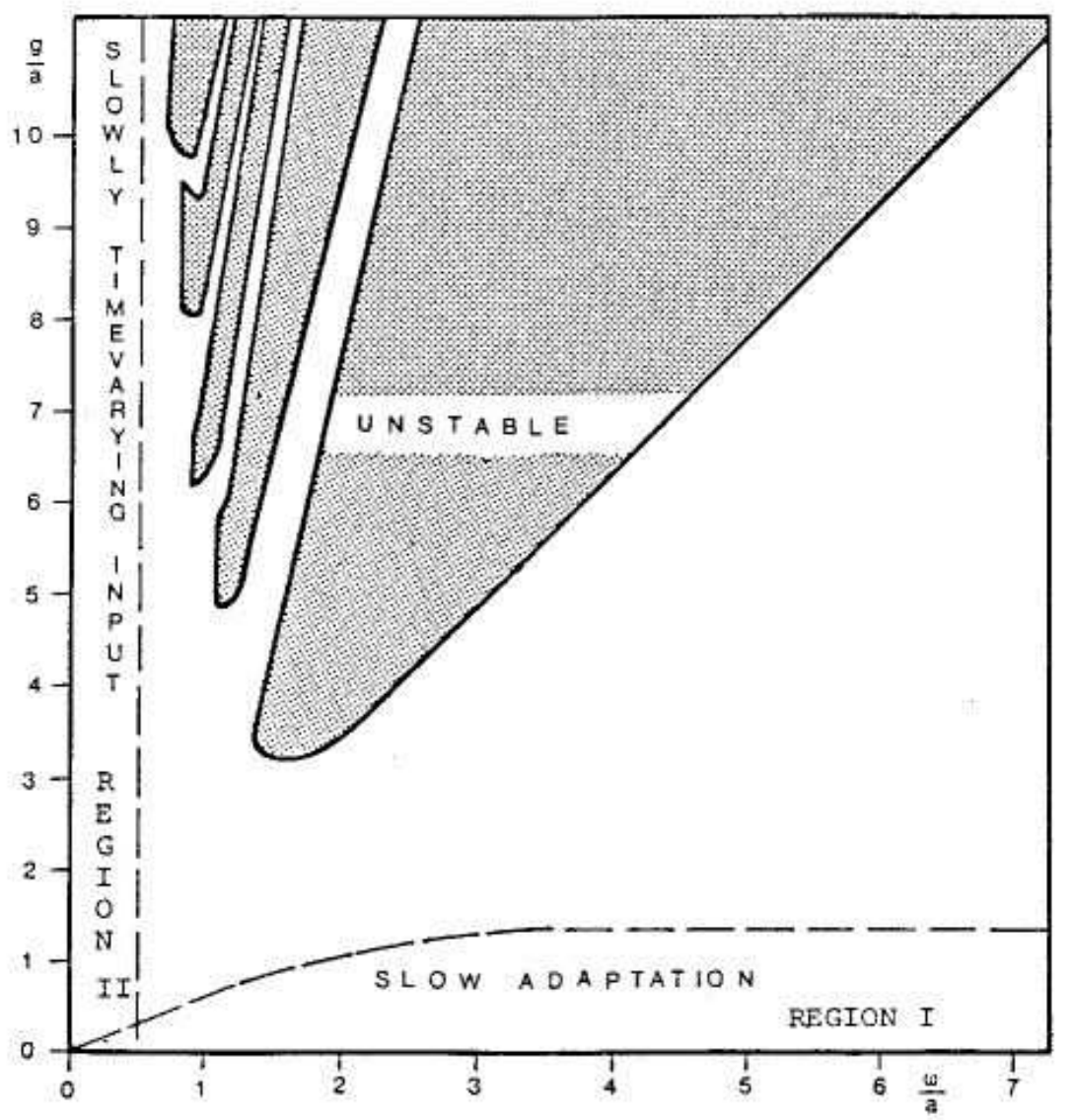

FIG. 4. MIT Rule Performance for first order plant with sinusoidal input

Suppose first that $r(t)=R$, a constant. Then the MIT Rule (3) leads to a characteristic equation

$$
s+g k_{m} k_{p} R^{2} Z_{p}(s)=0 .
$$

Figure 4 indicates no instability will arise for $Z_{p}(s)=(s+1)^{-1}$, but the seeds of an instability can be seen in this equation. If $Z_{p}(s)$ has a right half plane zero, then for high enough $g R^{2}$, an instability will occur. Observe that in some sense, the adaptation time scale is colliding with the time scale of the plant dynamics. If $Z_{p}(s)$ is of the form $\left(s^{2}+a s+b\right)^{-1}$ then again instability will occur if $g k_{m} k_{p} R^{2}>a b$. Again, one could say that the adaptation time scale is colliding with the time scale of the plant dynamics. Indeed for this example where the input is a constant, to ensure stability for all $g R^{2}$, one would need 
$\arg Z_{p}(j \omega) \in(-\pi / 2, \pi / 2) \quad \forall \omega \in \mathbb{R}$

If instead of a constant input one has a sinusoidal input, it is not possible to perform as simple an analysis. The differential equation for $k_{c}$ is actually a Mathieu equation. Instability corresponds to a sort of resonance effect which can only arise when $g$ exceeds a certain threshold. This is consistent with the fact that in Figure 4 , there are no instabilities for any $\omega$ provided $g$ is small enough. However, the mere labelling of the phenomenon as a Mathieu equation helps little in yielding an understanding, especially a level of understanding that would be readily transferable to use of the MIT rule with other plants.

Below, we will develop further some of the above ideas. But, we first need to explain a second instability mechanism which arises when one adjusts the problem statement in a more practical direction. With plant $k_{p} Z_{p}(s)$, we have assumed that all the unknownness is in $k_{p}$. However, we can also suppose there is some unknownness (e.g. unmodelled high frequency dynamics) in $Z_{p}(s)$, and that our knowledge of $Z_{p}(s)$ is captured by a model transfer function $Z_{m}(s)$, which is like $Z_{p}(s)$, but not identical. The error signal we construct is shown in Figure 5. The same rule (2) is still adopted. The expanded form of (3) is now

$$
\dot{k}_{c}=-g\left[Z_{m}(s) k_{m} r(t)\right]\left[Z_{p}(s) k_{p} k_{c} r(t)\right]+g\left[Z_{m}(s) k_{m} r(t)\right]^{2} .
$$

[The notation is of course suggestive, though not strictly proper.]

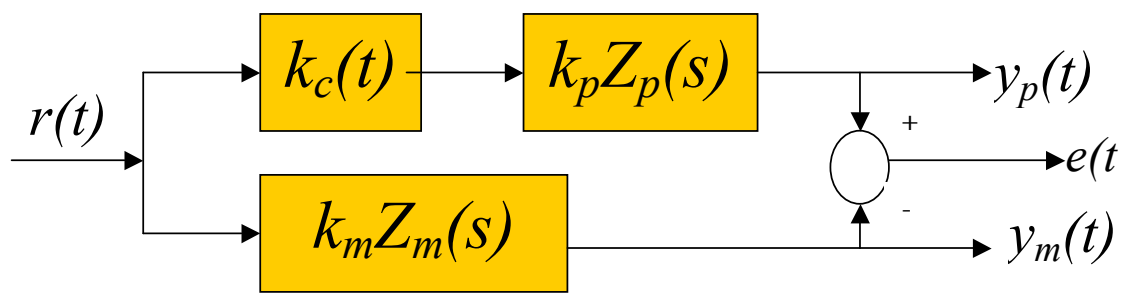

FIG. 5. MIT Rule Set-up with Model distinct from Plant

One might argue that the MIT rule, being an adaptive rule, is meant to cope with uncertainties or inaccuracies and that there should therefore be some capability to deal with $Z_{p}(s)$ unequal to $Z_{m}(s)$. Figure 6 shows what happens; the regions of gainfrequency pairs giving instability have expanded, and while there is still protection at low frequencies, at high frequencies instability is guaranteed for any gain, no matter how small. 


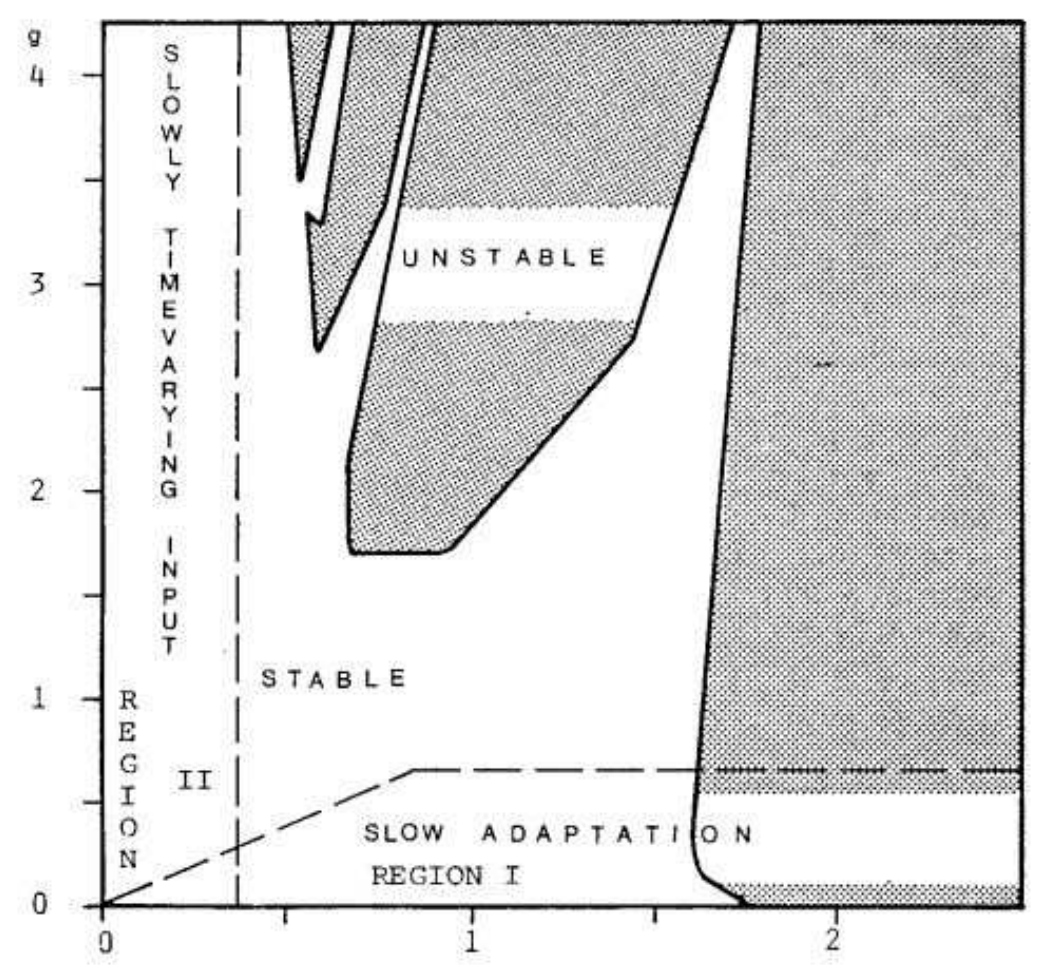

Fig. 6. MIT Rule Performance with Model distinct from Plant

These phenomena can be understood with a tool called averaging theory; references [2] and [5] contain much material applying averaging theory to adaptive control. Averaging theory is a tool which is usable given separation of time scales of the closedloop plant dynamics and the learning/adaptation rate. It indicates that if in (6) the gain $g$ is small enough so that $\dot{k}$ is small, then the behaviour of (6) can be approximated by the behaviour of

$$
\dot{k}_{c}^{*}=-g\left[Z_{m}(s) k_{m} r(t)\right]\left[Z_{p}(s) k_{p} r(t)\right] k_{c}^{*}(t)+g\left[Z_{m}(s) k_{m} r(t)\right]^{2} .
$$

In $(6) k_{c}(t)$ is processed by the dynamics of $Z_{p}(s)$, before contributing on the right hand side to making up $\dot{k}_{c}$. In (7), this is not the case. As further intuition, note that $k_{p}$, which is constant, can be pulled out of the action on it by $Z_{p}(s)$; it is reasonable then that if $k_{c}$ is nearly constant, the same conclusion remains true for it.

Now stability in (7) is assured if the average value of

$$
a(t):=\left[Z_{m}(s) k_{m} r(t)\right]\left[Z_{p}(s) k_{p} r(t)\right]
$$


is positive. Since $k_{m}$ and $k_{p}$ are constant with the same sign, it is clear that we need $r(t)$ to have the bulk of its energy confined to those frequencies where $Z_{m}(s)$ and $Z_{p}(s)$ have similar frequency responses. Obviously, if $Z_{p}(s)=\exp (-s) Z_{m}(s)$, and $r(t)=\sin \omega t$, then for $\omega$ suitably large, $a(t)$ in (8) will have a negative average value; this accounts for the high frequency behaviour in Figure 6 .

The MIT rule ideas, with the precautionary interpretations above, have been used for the adaptive servo control of large antennas, such as antennas for radio astronomy. The adaptive control problem is remarkably similar, and its satisfactory resolution requires separation of the time scales, [12].

4. Bursting. In the early 1980s, scattered reports appeared of adaptive control systems which worked well for a long period, say a week, and then unexpectedly burst into an oscillation which then died away. In this section, we describe the reasons for the phenomenon, and indicate how such undesirable behaviour can be avoided.

Figure 7 shows the phenomenon; an adaptive controller is connected to a first order plant, and set-point control is sought.

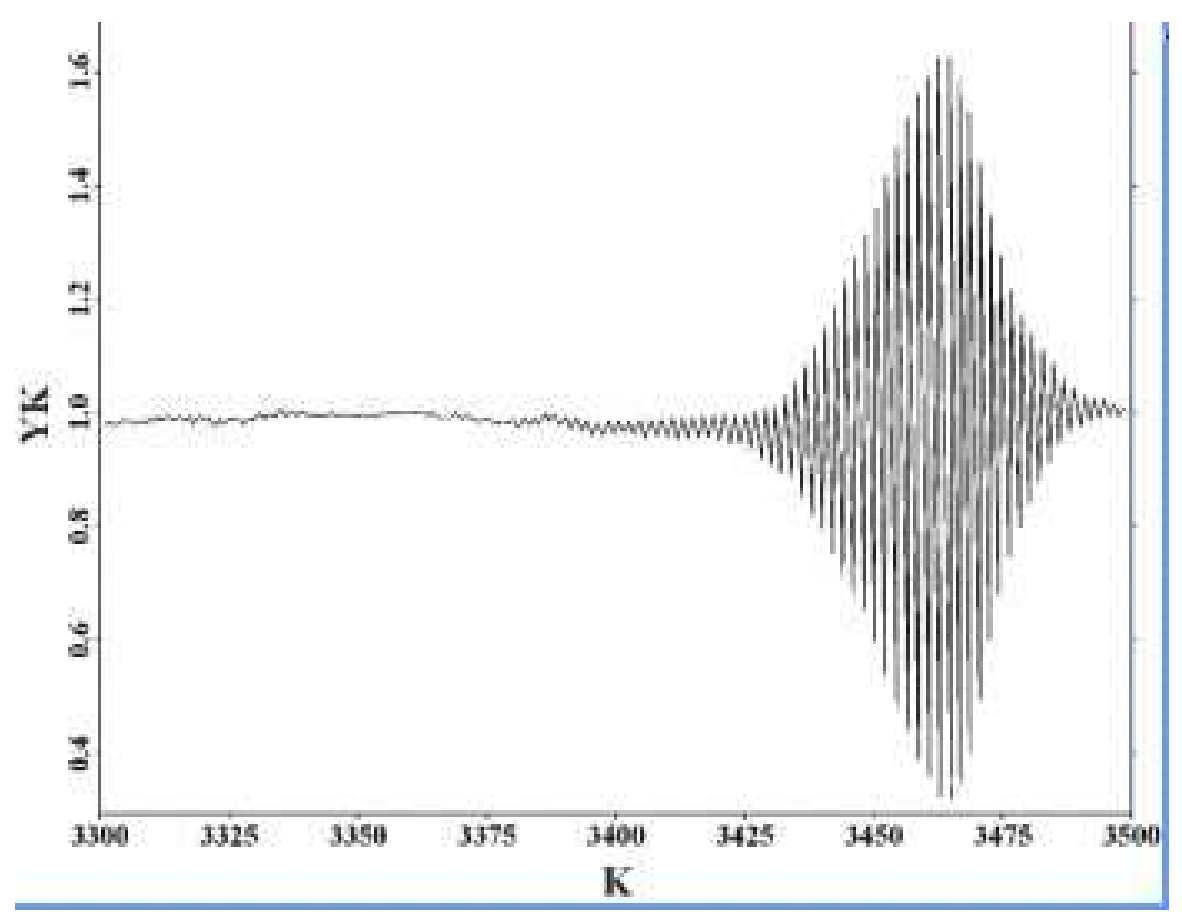

FIG. 7. Plant Output with Adaptive Controller connection during a "Burst"

The plant in question is described by a differential equation

$$
\dot{y}+c y=b u
$$


for some constant, unknown $b$ and $c$. The identifier block in Figure 2 has the task of using the measurements of $u$ and $y$ to determine $b$ and $c$. Now with most measurements this is possible; but if $u$ is a nonzero constant (as expected with set-point control), the identifier can be expected to identify the plant $\mathrm{DC}$ gain, i.e. the ratio $b / c$, but not to identify $b$ and $c$ separately. However the identifier does not know that the input is such that it cannot identify $b$ and $c$ separately; it simply runs an algorithm driven by $u$ and $y$ and producing quantities $\hat{b}$ and $\hat{c}$, estimates of $b$ and $c$, which are supposed to have the property that $\hat{b} \rightarrow b$ and $\hat{c} \rightarrow c$ as time evolves.

A typical identifier by the way has the form

$$
\left[\begin{array}{c}
\dot{\hat{b}} \\
\dot{\hat{c}}
\end{array}\right]=\left[\begin{array}{c}
\text { time function derived from } u, y \\
\text { second time function derived from } u, y
\end{array}\right]
$$

and the error obeys

$$
\frac{d}{d t}\left[\begin{array}{l}
b-\hat{b} \\
c-\hat{c}
\end{array}\right]=-\left[\begin{array}{l}
u \\
y
\end{array}\right]\left[\begin{array}{l}
u \\
y
\end{array}\right]\left[\begin{array}{c}
b-\hat{b} \\
c-\hat{c}
\end{array}\right] .
$$

In case $u$ and $y$ are both constant, the equation then implies

$$
u(\hat{b}-b)+y(\hat{c}-c) \rightarrow 0
$$

while also $c y=b u$. It follows that $\hat{b} / \hat{c} \rightarrow b / c$.

With constant $u$ and $y$ the actual time trajectories followed by $\hat{b}$ and $\hat{c}$ will depend on initial conditions, drift, noise etc. In any case, the adaptive controller uses $\hat{b}$ and $\hat{c}$ to implement a control law. Suppose that the controller simply inserts a gain $K$ with a view to having a high design bandwidth $d=c+b K$. So it will choose $\hat{K}=(d-\hat{c}) / \hat{b}$ and the actual closed loop pole will be at $-c-b \hat{K}$. If $\hat{b} / \hat{c}=b / c$, this pole is then at $-b d / \hat{b}$. Since $\hat{b}$ and $\hat{c}$ are not separately constrained but can move round, randomly in many cases, the situation can arise that $-b d / \hat{b}>0$; instability then occurs. But with instability comes richer signals, and much improved identification. Much improved identification then produces a stabilizing controller, and the whole set-up recovers, until the next time the drifting signals induce an unstable closed loop. This explanation of the bursting phenomenon can be found in [13]

Obviously, one wants $\hat{b}-b$ and $\hat{c}-c$ to go to zero, preferably exponentially fast,to give protection against noise etc. It is nontrivial that a sufficient and virtually necessary condition for this is that for some $\alpha_{1}, \alpha_{2}$ and $T$ all positive and for all $s$, there holds

$$
\alpha_{1} I<\int_{s}^{s+T}\left[\begin{array}{l}
u(\sigma) \\
y(\sigma)
\end{array}\right][u(\sigma) y(\sigma)] d \sigma<\alpha_{2} I .
$$


This is termed a persistency of excitation condition. Such a condition was advanced simultaneously by several workers; the simplest proof is probably to be found in [14].

Condition (13) is far from straightforward to apprehend. It uses signals internal to the loop. Alternative conditions have been found, starting with [15], which are conditions on external signals and as such are much easier to verify or arrange to have fulfilled.

If we require the external (reference) signal to be a sum of sinusoids (regarding a constant signal as a zero frequency sinusoid), then for this problem (13) is guaranteed by requiring that there be at least one complex sinusoid; more generally, if the plant has $m-1$ finite zeros and $n$ poles (thus there are $m+n$ coefficients in the numerator and denominator polynomials of the transfer function to be identified), then there must be an external input signal that excites $m+n$ distinct frequencies (with 0 counting as one frequency and $\pm j \omega$ counting as two frequencies). Such a condition ensures that the generalization of (13) is fulfilled, which in turn means that the adaptive identifier can learn each unknown parameter. Of course, broadband noise will also qualify as a rich enough input signal.

The fact that successful identification requires rich enough signals goes back earlier than the work on bursting, see [16], but the relevance of the observation to explain the bursting phenomenon was not appreciated until much later.

As a practical issue then, set-point control and adaptation are incompatible. Remedies include turning off the adaptation where the input is constant, or superimposing a (presumably small) rich excitation on the external set-point signal to ensure the adaptation does not lead to bursting.

5. The Rohrs' Counterexample. The Rohrs' counterexample amounted to an ideological controversy of the 1980s. We note the two principal journal references, [17, 18]. Broadly speaking, one set of proponents argued that because so many adaptive control algorithms postulated positive realness of a certain transfer function derived from the plant, and because a real-world plant had unmodelled dynamics which would force the relative degree of its transfer function to exceed one (and thereby prevent the positive realness of certain transfer functions derived from it), then no adaptive control algorithm could work. Experiments were advanced to back up the conclusion. To begin with, the reasoning was illogical; first, the postulation of positive realness was a sufficient but not necessary condition for the validity of the theorems asserting convergence of the algorithms, as they were published, so it did not follow that in the absence of positive realness a disaster would have to occur when these algorithms were used. Second, no worker had ever said that for all conceivable algorithms, one would have to have a positive real condition, even as a sufficient condition; so even if it had been established that none of the algorithms covered by the then-available 
theorems could work, i.e. yield convergent behaviour, there was no disproof of the existence of some other hitherto undiscovered algorithm which would work. Third, it was crystal clear to everyone that there were indeed successful applications of adaptive control, despite the jeremiadic cry. The ideological claim needed to be tempered, to one asserting the inapplicability of the theory in many circumstances.

By the time of the appearance of [17], much but perhaps not all of this tempering had occurred: "Our conclusion is that the adaptive algorithms as published in the literature are likely to produce unstable control systems if they are implemented on physical systems directly as they appear in the literature. The conclusions stem from the results of this paper which show unstable behavior of adaptive systems when these systems are confronted with two premises that cannot be ignored in any physical control design: 1) there are always unmodeled dynamics at sufficiently high frequencies (and it is futile to try to model these dynamics: and 2) the plant cannot be isolated from unknown disturbances (e.g., $60 \mathrm{~Hz}$ hum) even though these may be small."

What though of the experiments which justified the conclusion? For the most part, these were pointers to the inadequacies of the theory, though not quite the inadequacies asserted in [17]. Problems typically occurred in one or both of two situations. In the first situation, the reference signal comprised a step, which meant that it was not persistently exciting. The instability observed was therefore nothing other than bursting, as described in the previous section. In the second situation, a sinusoidal excitation was used, the frequency of which intruded into the region where there were significant unmodeled (and unmodelable) dynamics (By unmodelable dynamics, we mean that the number of parameters in the model of the real plant was too small to allow the model to capture all the dynamics present in the true plant). The situation was then very much like that discussed in the section on the MIT rule, where the second instability mechanism was described, and attributed to inability to properly model the true plant, coupled with having significant excitation in the frequency band where that ineffective modeling was occurring. These sorts of observations can be found in [18].

6. Iterative Control and Identification. A frequently advanced approach to adaptive control design is iterative identification and controller redesign, see e.g. $[19,20,21]$.

This is a form of adaptive control in which the tasks of identification and control are strictly separated. There is usually an underlying performance index which is to be minimized. One iteration comprises: (a) identifying the plant with the current controller (b) redefining the controller on the basis of the identified model of the plant and in order to minimize the performance index, and then implementing the new controller on the real plant. At this point, the identification task is re-commenced, 
with the old information being (largely at least) thrown away. If the plant is unknown initially, but constant, then one expects convergence, i.e. the controller settles down. However, if the plant is slowly varying, this will not happen, naturally. Furthermore, in the event that the modelling of the plant is based on using fewer parameters than is appropriate, the controller may settle down to an incorrect value (i.e. the performance index is not minimized), or it may be impossible to secure bounded signals in the loop, see [22].

While appealing conceptually, the above approach can lead to instability of the closed loop. In the remainder of this section, we shall explain why this is so.

At each iteration, there is delivered a model of the plant (i.e. the output of the identification process). We would term it a good model if a simulation of the model with a copy of the current controller behaves like the plant connected with the current controller; normally, we would expect the identification step to yield a good model. At this point, the algorithm changes the controller to better reflect the control objective. The controller change is determined by working with the current plant model, but with the new controller being attached to the actual plant rather than the model. One knows that the current controller connected to the plant will behave like the current controller connected to the (current) model. One wants the new controller connected to the plant to behave like the new controller connected to the current model.

This will not necessarily happen. Consider two transfer functions, a model transfer function $P_{1}=(s+1)^{-1}$ and a plant transfer function $P_{2}=(s+1)(0.1 s+1)^{-1}$. Consider Figure 8 and 9 . Figure 8 shows the open-loop step responses (i.e. the responses with a zero controller). Figure 9 shows the responses with two different constant gain controllers. It is evident that with gain 100, the closed-loop responses are very different.

The conclusion is that a model may be a good model of the plant with one controller, but it is not guaranteed to be a good model of the plant for all controllers. Therefore, if it is used as a basis for controller redesign, one even has the risk that a new controller, while fine with the model, could destabilise the plant.

There are at least two approaches to deal with this problem. One, due to Safonov and colleagues, e.g. $[23,24]$ is able to certify, for a wide class of controllers and despite the fact that the model is not identical with the plant, that insertion of the replacement controller will not produce closed-loop instability. The second uses gap metric ideas to identify controller changes which are small enough to not cause instability. If the iterative design calls for a big controller change, one moves from the current controller "in the direction of" but not all the way to the newly designed controller, i.e. one makes a "safe change", or one which will not induce instability, though it does improve performance, see e.g. [20, 21, 25, 26].

This idea has also been used in Iterative Feedback Tuning (an adaptive control procedure involving direct tuning of controller parameters), [27] and in Virtual Refer- 


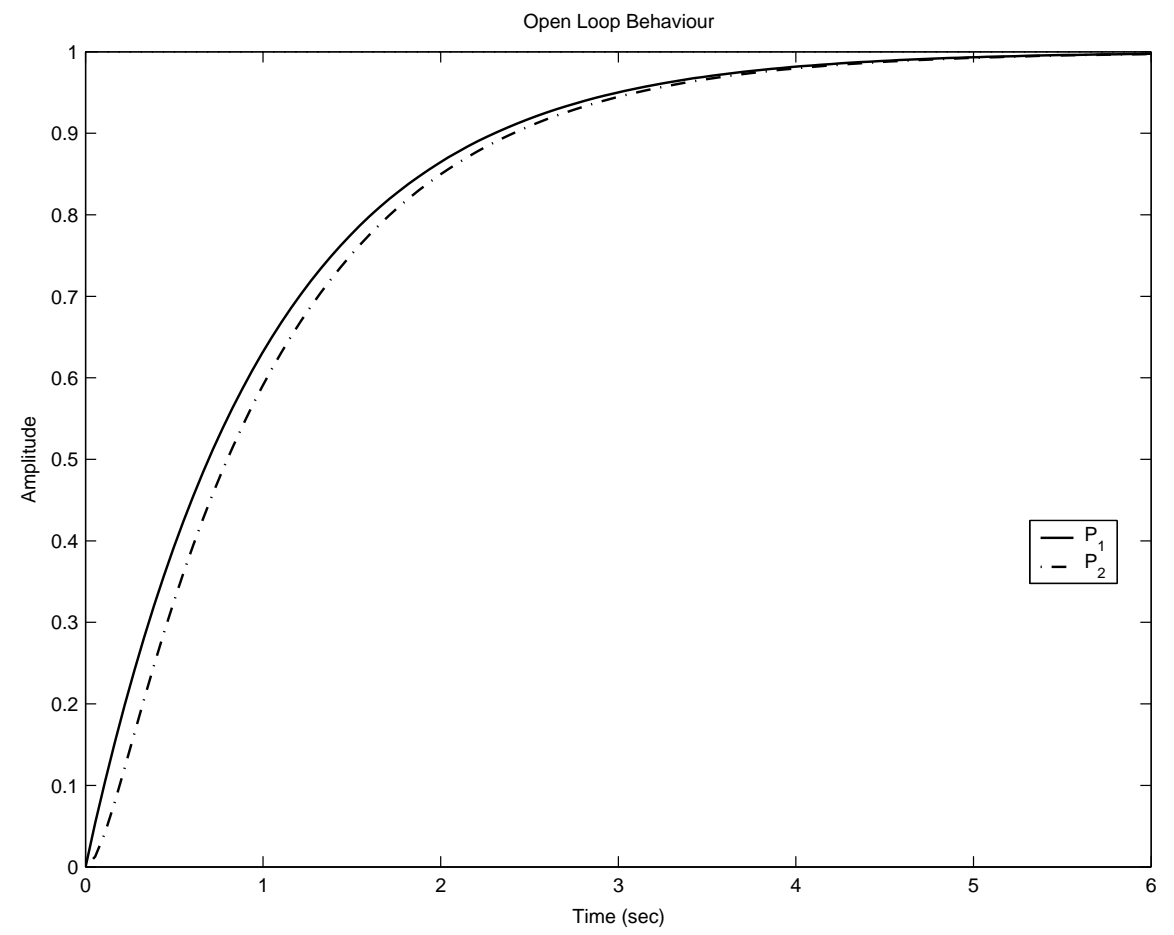

FIG. 8. Comparison of Plant and Model Responses in Open Loop

ence Feedback Tuning (another adaptive control procedure, especially aimed at model matching problems), [28].

The windsurfing approach to adaptive control [11] (which is an iterative identification and controller redesign approach) is also a very good example; at each controller re-design step, one expands the closed-loop bandwidth by a small amount, which is consistent with making a safe and small controller change. The closed-loop bandwidth is expanded out until a bandwidth corresponding to the design objective is attained, or the algorithm indicates that, because of the identification uncertainty, there can be no guarantee that a further bandwidth expansion is safe, and pursuit of a wider closed-loop bandwidth should be abandoned.

This notion of having a flag in an adaptive control algorithm to indicate the inappropriateness of an originally posed objective is practically important, and missing from older adaptive control literature. Logic really demands it. If a plant is initially unknown or only partially unknown, a designer may not know a priori that a proposed design objective is or is not practically obtainable for the plant. Having the algorithm discover this is helpful.

7. Multiple Model Adaptive Control. Imagine a bus on a city street. The equations of motion have parameters which depend on the load, and the friction 


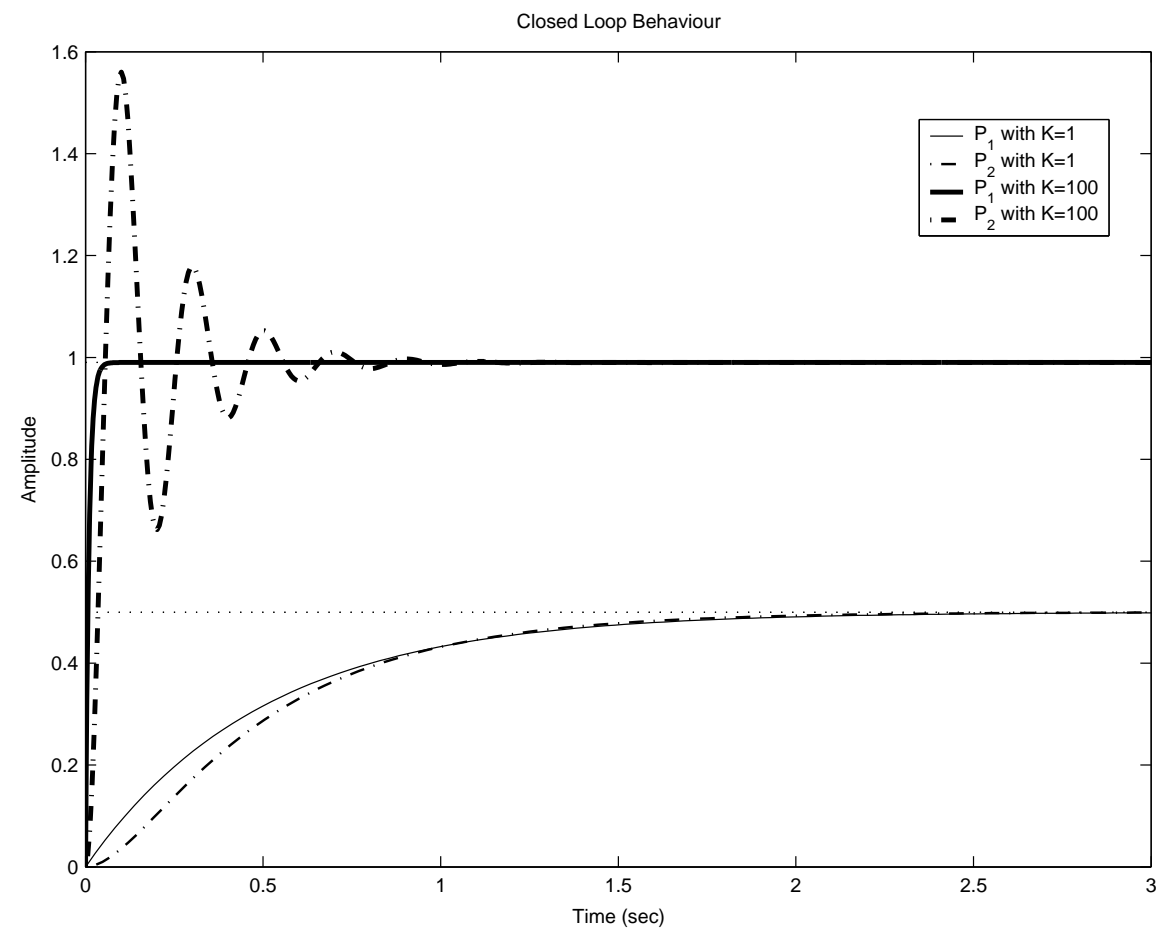

FIG. 9. Comparison of Plant and Model Responses in Closed Loop

between the tyres and the road. The friction coefficient will depend on the road surface, including the amount of oil and/or water on the surface. This is an example of a plant which may have a complicated transfer function, or even a non-linear description, while also containing a (frequently small) number of physical parameters which are unknown and/or changeable. Call such a plant $P(\lambda)$ where $\lambda$ is the vector of plant parameters.

It would be nice if one could learn $\lambda$ from measurements by some equations such as

$$
\dot{\hat{\lambda}}=f(\hat{\lambda}, \text { measurements })
$$

with $\hat{\lambda} \rightarrow \lambda_{\text {true }}$. This may however be too hard, especially for nonlinear plants, other than in some specific cases.

A completely different approach, multiple model adaptive control, has been suggested to cope with this situation see e.g. [29, 30, 31]. These references are all for problems with a linear plant; it is clear that the methodology and architecture implicit in the remarks to now and indeed in the next several paragraphs in principle could apply to nonlinear plants also, though the details of implementation may be much harder.

Suppose the unknown parameter vector $\lambda$ lies in a bounded closed simply-connect- 
ed region $\Lambda$ with $\bar{P}$ the associated true plant. Choose a representative set of values $\lambda_{1}, \lambda_{2}, \ldots, \lambda_{N}$ in the region, with associated plants $P_{1}, P_{2}, \ldots P_{N}$. Design $N$ controllers $C_{i}$ such that $C_{i}$ gives good performance with $P_{i}$ and plants "near" $P_{i}$.

The adaptive control algorithm works as follows. With one controller connected, run an algorithm which estimates at any time instant the particular representative model from $P_{1}, P_{2}, \ldots P_{N}$, call it $P_{I}$, which is the best model to explain the measurements of the inputs and outputs of $\bar{P}$. Then connect up $C_{I}$. Hopefully after at most a finite number of switchings, the best controller for the plant is obtained, and indeed the thrust of early theoretical results on multiple model control was to establish conclusions such as this, at least for linear plants and controllers.

There are problems with this intuitively appealing framework at two levels:

- How many plants $P_{i}$ should be chosen, how does one choose a representative set of plants $P_{1}, P_{2}, \ldots P_{N}$, and how can it be assured that the controllers $C_{i}$ will give good performance for plants near $P_{i}$ ? (Indeed, what does "near" mean?)

- If controller $C_{J}$ is connected, and it turns out that $P_{I}$ is the best explainer of $\bar{P}$ (a good model), there is no guarantee that after switching in of $C_{I}$ to replace $C_{J}, P_{I}$ will continue to be a good model of $\bar{P}$. Instead, $P_{K}$ for some other index $K$ might be a good model. (This point was explained in the previous section).

We postpone for several paragraphs dealing with the first issue. How can the second be resolved? The situation is analogous to that for iterative identification and control. First, instability may be encountered if the basic algorithm is used as for iterative identification and control. Second, for broad classes of controllers, before a particular $C_{i}$ is switched in, the methods of Safonov et al, see e.g. [23], allow prospective evaluation of its suitability, including closed-loop stability, at least if the controller is minimum phase. Third, one can conceive of safe switching, i.e. depending on the quality of the identification of the loop comprising the current controller and true plant, one can determine which controllers are safe to switch in, and one can elect to switch in one of them, only when it is foreshadowed that were the true plant replaced by the best model of it, the proposed new controller would offer superior performance to the current controller.

For linear plants and controllers, the algorithms of [29, 30] can indeed be modified along these lines [32], and safe switching results, with the penalty that switching occurs less frequently than with the unmodified algorithm (due to certain proposed switching being ruled out on safety grounds). This is a reasonable penalty to pay, given that the unmodified algorithm on occasions gives rise to connection of a destabilising controller.

How now can we deal with the first issue? This problem is addressed in [33] for linear plants. In outline, one sequentially picks $P_{1}, P_{2}, \ldots P_{N}$ by a systematic procedure. Choose $\lambda_{1}$ and thus $P_{1}$ arbitrarily. Design $C_{1}$. Now determine an open ball $B_{1}$ 
around $P_{1}$ for which $C_{1}$ constitutes a satisfactory design. [One approach is to choose $P$ to be in the ball if and only if $\delta_{\nu}\left(P, P_{1}\right)<0.3 b_{P_{1}, C_{1}}$. Here $\delta_{\nu}$ denotes the $\nu$-gap metric distance between $P$ and $P_{1}$, and $b_{P_{1}, C_{1}}$ is the generalised stability margin, viz.

$$
b_{P_{1}, C_{1}}=\left\|T\left(P_{1}, C_{1}\right)\right\|_{\infty}^{-1}
$$

with

$$
T_{P_{1}, C_{1}}=\left[\begin{array}{c}
P_{1} \\
I
\end{array}\right]\left[I-C_{1} P_{1}\right]^{-1}\left[-C_{1} I\right] .
$$

Now choose a $\lambda_{2}$ near the outer limit of the ball $B_{1}$, and design $C_{2}$ using $P_{2}=$ $P\left(\lambda_{2}\right)$. Determine an open ball $B_{2}$ around $P_{2}$ for which $C_{2}$ is a good controller. Choose $\lambda_{3}$ near the outer limit of $B_{1} \cup B_{2}$, and so on. Of course, the $\lambda_{i}$ must be drawn from $\Lambda$. The procedure terminates at some finite $N$ (by the Heine-Borel Theorem). Note that the difficulty for nonlinear plants is the absence of practical ways to generalise the $\nu$-gap metric distance between $P$ and $P_{1}$ and the generalised stability margin, which are necessary in the linear system case for a quantitative version of the in-principle algorithm.

In [33], a plant collection is considered where there are two scalar parameters that can vary, a gain and a right-half-plane zero position. The scheme just proposed rapidly determines a collection of plants which turns out to be very similar to these obtained in [29], where a trial and error approach was used that must have been tedious.

8. Conclusions. Let us now summarise some of the key lessons from this survey of decades of adaptive control difficulties.

- The MIT rule confirms that keeping adaptation and plant dynamics time scales separate reduces the likelihood of problems. It also emphasises that one should model as well as possible, even if there is adaptive capability.

- The bursting study contains several messages. First, one should not use more parameters than one needs for modelling purposes; if the real plant were first order, and a second order model was used, trouble could be expected. Second, it also confirms that to learn, one needs satisfactory experimental conditions, where satisfactory means persistently rich excitations. In particular, if you want to keep learning, you need more excitation than a constant reference signal can provide.

- The iterative control re-design and identification study reminds us that a good model of a plant is only a good model for some controllers. This is also a message for multiple model adaptive control. Abrupt controller changes can introduce instability, even if the new controller is defined with what has been a good model. Safe adaptive control is one remedy. 
- For multiple model adaptive control, it is possible to pick representative models systematically, at least in the linear cases. Even then however, safe adaptive control procedures should be used, for the same reasons as in iterative control re-design and identification.

There is also a meta-lesson. The examples given, including the Rohrs' counterexample, exemplify a form of carelessness or overconfidence among theoreticians, who proposed algorithms without recognising that on occasions disastrous behaviour could occur. The disastrous behaviour was instability, (and there were very different reasons for it in the various examples).

Apart from the lesson that theoreticians need to be more cautious, what more might be said about future work? The stand-out issue seems to be to push out the MMAC ideas to non-linear adaptive control. The challenges include especially providing a sound basis for picking representative models, which is likely to require some form of $\nu$-gap metric suitable for nonlinear systems. While such an entity has been defined, [34], the calculations would appear to be overpoweringly complicated.

Acknowledgments. This work was supported by an Australian Research Council Discovery Projects Grant and by National ICT Australia, which is funded by the Australian Government's Department of Communications, Information Technology and the Arts and the Australian Research Council through the Backing Australia's Ability initiative and the ICT Centre of Excellence Program.

\section{REFERENCES}

[1] GC Goodwin and KS Sin, Adaptive Filtering, Prediction and Control, Prentice Hall, Englewood Cliffs, NJ 1984.

[2] BDO Anderson, RR Bitmead, CR Johnson Jr, PV Kokotovic, RL Kosut, imy Mareels, L Praly, ANd BD Riedle, Stability of Adaptive Systems, MIT Press, 1986.

[3] KJ Astrom and B Wittenmark, Adaptive Control, Addison Wesley, 1989.

[4] KS Narendra and AM Annaswamy, Stable Adaptive Systems, Prentice Hall, 1989.

[5] S Sastry and M Bodson, Adaptive Control, Prentice Hall, 1989.

[6] M Krstic, I Kanellakopoulos, and P Kokotovic, Nonlinear and Adaptive Control Design, John Wiley, 1995.

[7] IMY Mareels and R R Bitmead, Non-linear dynamics in adaptive control: Periodic and chaotic stabilization, Automatica, 22(1986), pp. 641-655.

[8] IMY Mareels and R R Bitmead, Non-linear dynamics in adaptive control: Periodic and chaotic stabilization-II Analysis, Automatica, 24(1988), pp. 485-497.

[9] HP Whitaker, An Adaptive System for Control of the Dynamics Performances of Aircraft and Spacecraft, Inst Aeronautical Services, Paper 59-100, 1959.

[10] PV Osbourne, HP Whitaker, And A Kezer, New Developments in the Design of Model Reference Adaptive Control Systems, Inst Aeronautical Services, Paper 61-39, 1961.

[11] Imy Mareels, BDO Anderson, RR Bitmead, M Bodson, and S Sastry, Revisiting the MIT Rule for Adaptive Control, Proc 2nd IFAC Workshop on Adaptive Systems in Control and Signal Processing, 1986, pp. 161-166. 
[12] RJ Evans, imy Mareels, LJ Sciacca, DN Cooper, RH Middleton, RE Betz, and RA Kennedy, Adaptive servo control of large antenna structures, in: Model Identification and Adaptive Control, GC Goodwin, (Ed), Springer 2001, pp. 127-151.

[13] BDO Anderson, Adaptive systems, Lack of Persistency of Excitation and Bursting Phenomena, Automatica, 21(1985), pp. 247-258

[14] BDO Anderson, Exponential Stability of Linear Equations Arising in Adaptive Identification, IEEE Trans Auto Control, AC-22(1977), pp. 83-88.

[15] BDO Anderson and CR Johnson JR, Exponential Convergence of Adaptive Identification and Control Algorithms, Automatica, 18(1982), pp. 1-13.

[16] KJ Astrom And T Bohlin, Numerical identification of linear dynamic systems from normal operating records, in: Theory of Self-Adaptive Control Systems, PH Hammond, (Ed), Plenum, 1966.

[17] CE Rohrs, L Valavani, M Athans, and G Stein, Robustness of continuous-time adpative control algorithms in the presence of unmodeled dynamics, IEEE Trans Auto Control, 30(1985), pp. 881-889.

[18] KJ Astrom, A commentary on the C E Rohrs et al paper 'Robustness of continuous-time adaptive control algorithms in the presence of unmodeled dynamics', IEEE Trans Auto Control, 30(1985), 889.

[19] P Albertos, Model-based Iterative Control Design, in: Iterative Identification and Control, P Albertos and A Salas (Eds), Springer 2002, pp. 121-142.

[20] RR Bitmead, Iterative Optimal Control Design, in: Iterative Identification and Control, P Albertos and A Salas (Eds), Springer 2002, pp. 167-184.

[21] M Gevers, Identification and Validation for Robust Control, in: Iterative Identification and Control, P Albertos and A Salas (Eds), Springer 2002, 185-208.

[22] H Hulmarsson, S Gunnarsson, and M Gevers, Optimality and sub-optimality of iterative identification and control design schemes, Proc Amer Control Conf, Seattle, 1995, pp. 2559-2563.

[23] A Pail And MG SAfonov, Model reference adaptive control using multiple controllers and switching, Proc 42nd IEEE CDC, Hawaii, 2003, pp. 3256-3261.

[24] FB Cabral and MG Safonov, Unfalsified Model Reference Adaptive Control using the ellispoid algorithm, Proc 42nd IEEE CDC, Hawaii, 2003, pp. 3250-3255.

[25] Bdo Anderson and M Gevers, Fundamental Problems in Adaptive Control, in: Perspectives in Control, D Normand-Cyrot, Springer, Berlin, 1998, pp. 9-21.

[26] BDO Anderson, Windsurfing Approach to Iterative Control Design, in: Iterative Identification and Control P Albertos and A Salas (Eds), Springer 2002, pp. 143-166.

[27] F De Bruyne and LC Kammer, Iterative Feedback Tuning with guaranteed stability, Proc Amer Control Conf, 1999, pp. 3317-3321.

[28] A Lecchini, A Lanzon, And BDO Anderson, Safe adaptive controller changes based on reference model adjustments, Proc 42nd IEEE CDC, Hawaii, 2003, pp. 4297-4302.

[29] AS Monse, Supervisory Control of Families of Linear Set-point Controllers part 1: Exact Matching, IEEE Trans Auto Control, 41(1996), pp. 1413-1431.

[30] AS Monse, Control Using Logic-based Switching, in: Trends in Control: A European Perspective, A Isidori (ed), Springer, 1998, pp. 69-113.

[31] KS Narendra and J Balakrishnan, Adaptive Control Using Multiple Models, IEEE Trans Auto Control, 42(1997), pp. 171-187.

[32] JF Hespanha, D Liberzon, AS Morse, Bdo Anderson, tS Brinsmead, and F De Bruyne, Multiple Model Adaptive Control. Part 2: Switching, International Journal of Robust \& Nonlinear Control, 11(2001), pp. 479-496.

[33] Bdo Anderson, tS Brinsmead, F De Bruyne, J Hespanha, D Liberzon, and AS Morse, Multiple Model Adaptive Control. Part 1, Finite Controller Coverings, International Jour- 
nal of Robust and Nonlinear Control, 10(2000), pp. 909-929

[34] Bdo Anderson, TS Brinsmead, And F De Bruyne, The Vinnicombe Metric for Nonlinear Operators, IEEE Trans Auto Control, 47(2002), pp. 1450-1465. 